\title{
Explicit evaluation of quadratic Euler sums
}

\author{
Ce Xu* Yingyue Yang, Jianwen Zhang \\ School of Mathematical Sciences, Xiamen University \\ Xiamen 361005, P.R. China
}

\begin{abstract}
In this paper, we work out some explicit formulae for double nonlinear Euler sums involving harmonic numbers and alternating harmonic numbers. As applications of these formulae, we give new closed form representations of several quadratic Euler sums through Riemann zeta function and linear sums. The given representations are new.
\end{abstract}

Keywords Polylogarithm function; Euler sum; Riemann zeta function.

AMS Subject Classifications (2010): 11M06; 11M32; 33B15

\section{Introduction}

In this paper, harmonic numbers, alternating harmonic numbers and their generalizations define as

$$
H_{n}=\sum_{j=1}^{n} \frac{1}{j}, \zeta_{n}(k)=\sum_{j=1}^{n} \frac{1}{j^{k}}, L_{n}(k)=\sum_{j=1}^{n} \frac{(-1)^{j-1}}{j^{k}}, 1 \leq k \in \mathbb{Z} .
$$

The classical linear Euler sum is defined by

$$
S_{p, q}=\sum_{n=1}^{\infty} \frac{1}{n^{q}} \sum_{k=1}^{n} \frac{1}{k^{p}}
$$

where $p, q$ are positive integers, $q \geq 2$. In 1742, Goldbach proposed to Euler the problem of expressing the $S_{p, q}$ in terms of values at positive integers of the Riemann zeta function $\zeta(s)$. The Riemann zeta function and alternating Riemann zeta function are defined respectively by

$$
\zeta(s)=\sum_{n=1}^{\infty} \frac{1}{n^{s}}, \Re(s)>1 ; \bar{\zeta}(s)=\sum_{n=1}^{\infty} \frac{(-1)^{n-1}}{n^{s}}, \Re(s) \geq 1 .
$$

Euler showed this problem in the case $p=1$ and gave a general formula for odd weight $p+q$ in 1775. He conjectured that the double linear sums would be reducible to zeta values when $\mathrm{p}$ $+q$ is odd, and even gave what he hoped to obtain the general formula. In [3], D. Borwein, J.M. Borwein and R. Girgensohn proved conjecture and formula, and in [2], D.H. Bailey, J.M. Borwein and R. Girgensohn conjectured that the double linear sums when $p+q>7, p+q$ is even, are not reducible.

Let $\pi=\left(\pi_{1}, \ldots, \pi_{k}\right)$ be a partition of integer $p$ and $p=\pi_{1}+\cdots+\pi_{k}$ with $\pi_{1} \leq \pi_{2} \leq \cdots \leq \pi_{k}$.

\footnotetext{
${ }^{*}$ Corresponding author. Email: 15959259051@163.com
} 
The classical double nonlinear Euler sum of index $\pi, q$ is defined as follows (see [12])

$$
S_{\pi, q}=\sum_{n=1}^{\infty} \frac{\zeta_{n}\left(\pi_{1}\right) \zeta_{n}\left(\pi_{2}\right) \cdots \zeta_{n}\left(\pi_{k}\right)}{n^{q}}
$$

where the quantity $\pi_{1}+\cdots+\pi_{k}+q$ and $k$ called the weight and the degree.

In general, we can also define the double Euler sums by the series

$$
\sum_{n=1}^{\infty} \frac{\prod_{i=1}^{m_{1}} \zeta_{n}^{q_{i}}\left(k_{i}\right) \prod_{j=1}^{m_{2}} L_{n}^{l_{j}}\left(h_{j}\right)}{n^{p}}, \sum_{n=1}^{\infty} \frac{\prod_{i=1}^{m_{1}} \zeta_{n}^{q_{i}}\left(k_{i}\right) \prod_{j=1}^{m_{2}} L_{n}^{l_{j}}\left(h_{j}\right)(-1)^{n-1}}{n^{p}}
$$

where $p(p>1), m_{1}, m_{2}, q_{i}, k_{i}, h_{j}, l_{j}$ are positive integers. If $\sum_{i=1}^{m_{1}}\left(k_{i} q_{i}\right)+\sum_{j=1}^{m_{2}}\left(h_{j} l_{j}\right)+p=C(C$ is a positive integer), then we call the identity $C$ th-order Euler sums.

In [12], Philippe Flajolet and Bruno Salvy gave explicit reductions to zeta values for all linear sums

$$
\sum_{n=1}^{\infty} \frac{\zeta_{n}(p)}{n^{q}}, \sum_{n=1}^{\infty} \frac{L_{n}(p)}{n^{q}}, \sum_{n=1}^{\infty} \frac{\zeta_{n}(p)}{n^{q}}(-1)^{n-1}, \sum_{n=1}^{\infty} \frac{L_{n}(p)}{n^{q}}(-1)^{n-1}
$$

when $p+q$ is an odd weight. The evaluation of linear sums in terms of values of Riemann zeta function and polylogarithm function at positive integers is known when $(p, q)=(1,3),(2,2)$, or $p+q$ is odd $[6,12,18]$. For instance, we have

$$
\begin{gathered}
\sum_{n=1}^{\infty} \frac{H_{n}}{n^{3}}(-1)^{n-1}=-2 \operatorname{Li}_{4}\left(\frac{1}{2}\right)+\frac{11}{4} \zeta(4)+\frac{1}{2} \zeta(2) \ln ^{2} 2-\frac{1}{12} \ln ^{4} 2-\frac{7}{4} \zeta(3) \ln 2, \\
\sum_{n=1}^{\infty} \frac{L_{n}(1)}{n^{3}}(-1)^{n-1}=\frac{3}{2} \zeta(4)+\frac{1}{2} \zeta(2) \ln ^{2} 2-\frac{1}{12} \ln ^{4} 2-2 \operatorname{Li}_{4}\left(\frac{1}{2}\right) .
\end{gathered}
$$

The polylogarithm function defined for $|x| \leq 1$ by

$$
\operatorname{Li}_{p}(x)=\sum_{n=1}^{\infty} \frac{x^{n}}{n^{p}}, \Re(p)>1 .
$$

The relationship between the values of the Riemann zeta function and Euler sums has been studied by many authors, for example see [2-4,6-17,19-20].

From [18], for a multi-index $S=\left(s_{1}, s_{2}, \cdots, s_{k}\right)\left(s_{i} \in \mathbb{N}, s_{1} \geq 2\right)$, the multiple zeta value (MZV for short) and the multiple zeta-star value (MZSV for short) are defined, respectively, by convergent series

$$
\begin{gathered}
\zeta(S)=\zeta\left(s_{1}, s_{2}, \cdots, s_{k}\right)=\sum_{n_{1}>n_{2}>\cdots>n_{k} \geq 1} \frac{1}{n_{1}^{s_{1}} n_{2}^{s_{2}} \cdots n_{k}^{s_{k}}}, \\
\zeta^{\star}(S)=\zeta\left(s_{1}, s_{2}, \cdots, s_{k}\right)=\sum_{n_{1} \geq n_{2} \geq \cdots \geq n_{k} \geq 1} \frac{1}{n_{1}^{s_{1}} n_{2}^{s_{2}} \cdots n_{k}^{s_{k}}} .
\end{gathered}
$$

We put a bar on top of $s_{j}(j=1, \cdots k)$ if there is a sign $(-1)^{n_{j}}$ appearing in the denominator on the right. For example,

$$
\zeta^{\star}(\overline{2}, 3)=\sum_{m \geq n \geq 1} \frac{(-1)^{m}}{m^{2} n^{3}}=\sum_{m=1}^{\infty} \frac{(-1)^{m}}{m^{2}} \sum_{n=1}^{m} \frac{1}{n^{3}} .
$$


By using the above notations, we have

$$
\begin{gathered}
\zeta^{\star}(q, p)=S_{p, q}=\sum_{n=1}^{\infty} \frac{\zeta_{n}(p)}{n^{q}}, \zeta^{\star}(\bar{q}, p)=-\sum_{n=1}^{\infty} \frac{\zeta_{n}(p)}{n^{q}}(-1)^{n-1}, \\
\zeta^{\star}(q, \bar{p})=-\sum_{n=1}^{\infty} \frac{L_{n}(p)}{n^{q}}, \zeta^{\star}(\bar{q}, \bar{p})=\sum_{n=1}^{\infty} \frac{L_{n}(p)}{n^{q}}(-1)^{n-1} .
\end{gathered}
$$

The main purpose of this paper is to evaluate some quadratic Euler sums which involving harmonic numbers and alternating harmonic numbers, either linearly or nonlinearly. In this paper, we will prove that all quadratic double sums

$$
\sum_{n=1}^{\infty} \frac{H_{n}^{2}}{n^{2 m}}(-1)^{n-1}, \sum_{n=1}^{\infty} \frac{L_{n}^{2}(1)}{n^{2 m}}(-1)^{n-1}, \sum_{n=1}^{\infty} \frac{H_{n} L_{n}(1)}{n^{2 m}}(-1)^{n-1}, \sum_{n=1}^{\infty} \frac{H_{n} L_{n}(1)}{n^{2 m}}, \sum_{n=1}^{\infty} \frac{L_{n}^{2}(1)}{n^{2 m}}
$$

are reducible to polynomials in zeta values and to linear sums, $m$ is a positive integer. Similarly, we have the following relations

$$
\begin{gathered}
\sum_{n=1}^{\infty} \frac{H_{n}^{2}}{n^{2 m}(-1)^{n-1}}=-2 \zeta^{\star}(\overline{2 m}, 1,1)+\zeta^{\star}(\overline{2 m}, 2), \sum_{n=1}^{\infty} \frac{L_{n}^{2}(1)}{n^{2 m}}(-1)^{n-1}=-2 \zeta^{\star}(\overline{2 m}, \overline{1}, \overline{1})+\zeta^{\star}(\overline{2 m}, 2), \\
\sum_{n=1}^{\infty} \frac{L_{n}^{2}(1)}{n^{2 m}}=2 \zeta^{\star}(2 m, \overline{1}, \overline{1})-\zeta^{\star}(2 m, 2), \sum_{n=1}^{\infty} \frac{\zeta_{n}^{2}(p)}{n^{2 m}}=2 \zeta^{\star}(2 m, p, p)-\zeta^{\star}(2 m, 2 p), \\
\sum_{n=1}^{\infty} \frac{H_{n} L_{n}(1)}{n^{2 m}}=-\zeta^{\star}(2 m, \overline{1}, 1)-\zeta^{\star}(2 m, 1, \overline{1})+\zeta^{\star}(2 m, \overline{2}), \\
\sum_{n=1}^{\infty} \frac{H_{n} L_{n}(1)}{n^{2 m}}(-1)^{n-1}=\zeta^{\star}(\overline{2 m}, \overline{1}, 1)+\zeta^{\star}(\overline{2 m}, 1, \overline{1})-\zeta^{\star}(\overline{2 m}, \overline{2}) .
\end{gathered}
$$

The following lemma will be useful in the development of the main theorems.

Lemma 1.1 Let $n$ be a positive integers. We have the following relations

$$
\begin{gathered}
\sum_{k=1}^{n-1} \frac{L_{k}(1)}{n-k}(-1)^{k-1}=2(-1)^{n-1} \sum_{k=1}^{n} \frac{H_{k}}{k}(-1)^{k-1}-2(-1)^{n-1} L_{n}(2), \\
\sum_{m=n+1}^{\infty} \frac{L_{m}(1)}{m-n}(-1)^{m-n}=\sum_{k=1}^{n} \frac{H_{k}}{k}(-1)^{k-1}-\sum_{j=1}^{\infty} \frac{L_{j}(1)}{j}(-1)^{j-1}, \\
(-1)^{n-1} \sum_{k=1}^{n-1} \frac{L_{k}(1)}{n-k}=\sum_{k=1}^{n-1} \frac{H_{k}}{n-k}(-1)^{k}, \\
\sum_{m=n+1}^{\infty} \frac{H_{m}}{m-n}(-1)^{m-n}=\frac{L_{n}^{2}(1)+\zeta_{n}(2)}{2}-\ln 2\left(H_{n}+L_{n}(1)\right)-\sum_{n=1}^{\infty} \frac{H_{j}}{j}(-1)^{j-1} .
\end{gathered}
$$


where $\sum_{n=1}^{\infty} \frac{H_{n}(-1)^{n-1}}{n}=\frac{\zeta(2)-\ln ^{2} 2}{2}, \sum_{n=1}^{\infty} \frac{L_{n}(1)(-1)^{n-1}}{n}=\frac{\zeta(2)+\ln ^{2} 2}{2}$.

Proof. To prove (1.6), using Cauchy product formula, we have

$$
\frac{\ln (1+x)}{1-x}=\left(\sum_{n=1}^{\infty} \frac{(-1)^{n-1}}{n} x^{n}\right)\left(\sum_{n=1}^{\infty} x^{n-1}\right)=\sum_{n=1}^{\infty} L_{n}(1) x^{n} .
$$

Furthermore, by (1.10) and Cauchy product formula, we can obtain

$$
\frac{\ln ^{2}(1+x)}{1-x}=\sum_{n=1}^{\infty}(-1)^{n+1}\left(\sum_{k=1}^{n} \frac{L_{k}(1)}{n-k+1}(-1)^{k-1}\right) x^{n+1} .
$$

On the other hand, from [14], we get

$$
\ln ^{2}(1+x)=2\left\{\sum_{n=1}^{\infty} \frac{(-1)^{n-1}}{n^{2}} x^{n}-\sum_{n=1}^{\infty} \frac{H_{n}}{n}(-1)^{n-1} x^{n}\right\} .
$$

Similarly, applying (1.12), then have

$$
\frac{\ln ^{2}(1+x)}{1-x}=2 \sum_{n=1}^{\infty} \sum_{k=1}^{n}\left\{L_{n}(2)-\frac{H_{k}}{k}(-1)^{k-1}\right\} x^{n} .
$$

Comparing (1.11) and (1.13), we can obtain (1.6). Now, we prove (1.7). We see that we may rewrite the left side of (1.7) as

$$
\begin{aligned}
\sum_{m=n+1}^{\infty} \frac{L_{m}(1)}{m-n}(-1)^{m-n} & =\sum_{j=1}^{\infty} \frac{L_{n+j}(1)}{j}(-1)^{j} \\
& =\sum_{j=1}^{\infty} \frac{(-1)^{j}}{j}\left\{\sum_{k=1}^{j} \frac{(-1)^{k-1}}{k}+\sum_{k=j+1}^{n+j} \frac{(-1)^{k-1}}{k}\right\} \\
& =\sum_{j=1}^{\infty} \frac{L_{j}(1)}{j}(-1)^{j}+\sum_{k=1}^{n} \frac{(-1)^{k-1}}{k} \sum_{j=1}^{\infty}\left(\frac{1}{j}-\frac{1}{j+k}\right) \\
& =\sum_{k=1}^{n} \frac{H_{k}}{k}(-1)^{k-1}-\sum_{j=1}^{\infty} \frac{L_{j}(1)}{j}(-1)^{j-1} .
\end{aligned}
$$

Similarly to the proof of (1.6) and (1.7), we can prove (1.8) and (1.9).

\section{Main Theorems and Proof}

In this section, we will establish some explicit relationships which involve alternating quadratic Euler sums and linear sums.

Theorem 2.1 For integer $m \geq 1$, we have

$$
\sum_{j=1}^{m-1}(-1)^{j-1} \bar{\zeta}(m-j+1) \zeta^{\star}(j+1,1)+(-1)^{m-1} \ln 2 \zeta^{\star}(m+1,1)-\zeta^{\star}(\overline{m+2}, 1)
$$




$$
\begin{aligned}
& -(-1)^{m-1} \ln 2 \zeta^{\star}(\overline{m+1}, 1)-(-1)^{m-1} \sum_{n=1}^{\infty} \frac{(-1)^{n-1} H_{n} L_{n}(1)}{n^{m+1}} \\
& =\frac{1}{2} \sum_{n=1}^{\infty} \frac{(-1)^{n-1} H_{n}^{2}}{n^{m+1}}-\frac{1}{2} \zeta^{\star}(\overline{m+1}, 2)+\zeta(2) \bar{\zeta}(m+1) .
\end{aligned}
$$

Proof. Let

$$
I_{m}=\sum_{n=1}^{\infty} \frac{(-1)^{n-1}}{n^{m}(n+k)}
$$

Using the definition of $I_{m}$, we can easily verify that

$$
I_{m}=\sum_{j=1}^{m-1} \frac{(-1)^{j-1}}{k^{j}} \bar{\zeta}(m-j+1)+\frac{(-1)^{m-1}}{k^{m}} \ln 2+\frac{(-1)^{m+k}}{k^{m}} \ln 2-\frac{(-1)^{m+k}}{k^{m}} L_{k}(1) .
$$

On the other hand, we have

$$
\sum_{k=1}^{\infty} \frac{H_{k}}{k(n+k)}=\frac{1}{n}\left(\frac{1}{2} H_{n}^{2}+\frac{1}{2} \zeta_{n}(2)+\zeta(2)-\frac{H_{n}}{n}\right) .
$$

Multiplying (2.3) by $\frac{(-1)^{n-1}}{n^{m}}$ and summing with respect to $n$. The result is

$$
\sum_{n=1}^{\infty} \sum_{k=1}^{\infty} \frac{(-1)^{n-1} H_{k}}{k n^{m}(n+k)}=\frac{1}{2} \sum_{n=1}^{\infty} \frac{(-1)^{n-1} H_{n}^{2}}{n^{m+1}}-\frac{1}{2} \zeta^{\star}(\overline{m+1}, 2)+\zeta(2) \bar{\zeta}(m+1)+\zeta^{\star}(\overline{m+2}, 1) .
$$

Substituting (2.2) into (2.4) yields desired result.

Theorem 2.2 Let $s \geq 1$ be integer. Then the following identity holds

$$
\begin{aligned}
\frac{1}{2} \sum_{n=1}^{\infty} \frac{H_{n}^{2}}{n^{s}}(-1)^{n-1}-\sum_{n=1}^{\infty} \frac{H_{n} L_{n}(1)}{n^{s}}(-1)^{n-1}= & -\frac{3}{2} \zeta^{\star}(\bar{s}, 2)-s \zeta^{\star}(\overline{s+1}, 1)-\zeta^{\star}(s+1,1) \\
& -\sum_{j=2}^{s} \zeta^{\star}(j, 1) \bar{\zeta}(s+1-j)+\ln 2 \zeta^{\star}(\bar{s}, 1), \\
\frac{3}{2} \sum_{n=1}^{\infty} \frac{H_{n}^{2}-\zeta_{n}(2)}{n^{s}}=(s+1) \zeta^{\star}(s+1,1)- & \sum_{j=2}^{s-1} \zeta^{\star}(j, 1) \zeta(s+1-j), 2 \leq s \in Z .
\end{aligned}
$$

Proof. For real $-1 \leq x<1$ and integers $s(s \geq 2)$ and $t \geq 0$, consider function

$$
\begin{aligned}
T_{s, t}(x, y) & =\sum_{\substack{n, m=1 \\
n \neq m}}^{\infty} \frac{H_{n} x^{n} y^{m}}{n^{s} m^{t}(m-n)}=\sum_{\substack{n, m=1 \\
n \neq m}}^{\infty} \frac{H_{n} x^{n} y^{m}(m-n+n)}{n^{s} m^{t+1}(m-n)} \\
& =\sum_{\substack{n, m=1 \\
n \neq m}}^{\infty} \frac{H_{n} x^{n} y^{m}}{n^{s} m^{t+1}}+\sum_{\substack{n, m=1 \\
n \neq m}}^{\infty} \frac{H_{n} x^{n} y^{m}}{n^{s-1} m^{t+1}(m-n)}
\end{aligned}
$$




$$
\begin{aligned}
& =\sum_{n=1}^{\infty} \frac{H_{n} x^{n}}{n^{s}}\left(\sum_{m=1}^{\infty} \frac{y^{m}}{m^{t+1}}-\frac{y^{n}}{n^{t+1}}\right)+T_{s-1, t+1}(x, y) \\
& =\left(\sum_{n=1}^{\infty} \frac{H_{n} x^{n}}{n^{s}}\right) L i_{t+1}(y)-\left(\sum_{n=1}^{\infty} \frac{H_{n} x^{n} y^{n}}{n^{s+t+1}}\right)+T_{s-1, t+1}(x, y) .
\end{aligned}
$$

Define

$$
H_{s}^{(k)}(x)=\sum_{n=1}^{\infty} \frac{H_{n}^{k} x^{n}}{n^{s}},-1 \leq x<1,0 \leq k \in \mathbb{Z}, \Re(s) \geq 1 .
$$

Obviously, $H_{s}^{(0)}(x)=L i_{s}(x)$. Hence, we can rewrite (2.7) as

$$
T_{s, t}(x, y)=H_{s}^{(1)}(x) H_{t+1}^{(0)}(y)-H_{s+t+1}^{(1)}(x y)+T_{s-1, t+1}(x, y) .
$$

Telescoping this gives

$$
T_{s, t}(x, y)=\sum_{j=1}^{s} H_{j}^{(1)}(x) H_{s+t+1-j}^{(0)}(y)-s H_{s+t+1}^{(1)}(x y)+T_{0, s+t}(x, y) .
$$

Taking $t=0$ in (2.9) yield

$$
T_{s, 0}(x, y)=\sum_{j=1}^{s} H_{j}^{(1)}(x) H_{s+1-j}^{(0)}(y)-s H_{s+1}^{(1)}(x y)+T_{0, s}(x, y) .
$$

From the definition of $T_{s, t}(x, y)$, we conclude that

$$
\begin{aligned}
T_{s, 0}(x, y) & =\sum_{\substack{n, m=1 \\
n \neq m}}^{\infty} \frac{H_{n} x^{n} y^{m}}{n^{s}(m-n)}=\sum_{n=1}^{\infty} \frac{H_{n} x^{n}}{n^{s}} \sum_{\substack{m=1 \\
m \neq n}}^{\infty} \frac{y^{m}}{m-n} \\
& =\sum_{n=1}^{\infty} \frac{H_{n} x^{n} y^{n}}{n^{s}} \sum_{m=n+1}^{\infty} \frac{y^{m-n}}{m-n}-\sum_{n=1}^{\infty} \frac{H_{n} x^{n}}{n^{s}} \sum_{m=1}^{n-1} \frac{y^{m}}{n-m} \\
& =H_{s}^{(1)}(x y) H_{1}^{(0)}(y)-\sum_{n=1}^{\infty} \frac{H_{n} x^{n}}{n^{s}}\left(\sum_{j=1}^{n-1} \frac{y^{n-j}}{j}\right) . \\
T_{0, s}(x, y)= & \sum_{\substack{n, m=1 \\
n \neq m}}^{\infty} \frac{H_{n} x^{n} y^{m}}{m^{s}(m-n)}=\sum_{m=1}^{\infty} \frac{y^{m}}{m^{s}} \sum_{\substack{n=1 \\
n \neq m}}^{\infty} \frac{H_{n} x^{n}}{m-n} \\
= & -\sum_{m=1}^{\infty} \frac{x^{m} y^{m}}{m^{s}} \sum_{n=m+1}^{\infty} \frac{H_{n} x^{n-m}}{n-m}+\sum_{m=1}^{\infty} \frac{y^{m}}{m^{s}} \sum_{n=1}^{m-1} \frac{H_{n} x^{n}}{m-n} .
\end{aligned}
$$

Noting that

$$
\sum_{n=m+1}^{\infty} \frac{H_{n} x^{n-m}}{n-m}=\sum_{j=1}^{\infty} \frac{H_{n+j}}{j} x^{j}=\sum_{j=1}^{\infty} \frac{x^{j}}{j}\left(\sum_{k=1}^{j} \frac{1}{k}+\sum_{k=j+1}^{n+j} \frac{1}{k}\right)
$$




$$
\begin{gathered}
=\sum_{j=1}^{\infty} \frac{H_{j}}{j} x^{j}+\sum_{j=1}^{\infty} \frac{x^{j}}{j} \sum_{k=1}^{n} \frac{1}{k+j} \\
=H_{1}^{(1)}(x)+\sum_{k=1}^{n} \frac{1}{k} \sum_{j=1}^{\infty} x^{j}\left(\frac{1}{j}-\frac{1}{k+j}\right), \\
\sum_{j=1}^{n-1} \frac{H_{j}}{n-j}=H_{n}^{2}-\zeta_{n}(2), \sum_{k=1}^{n} \frac{H_{k}}{k}=\frac{H_{n}^{2}+\zeta_{n}(2)}{2} . \\
\lim _{x \rightarrow 1, y \rightarrow 1}\left\{H_{s}^{(1)}(x y) H_{1}^{(0)}(y)-H_{s}^{(1)}(x) H_{1}^{(0)}(y)\right\}=0, \\
\lim _{x \rightarrow 1, y \rightarrow 1}\left\{H_{s}^{(0)}(x y) H_{1}^{(1)}(x)-H_{s}^{(0)}(y) H_{1}^{(1)}(x)\right\}=0, \\
\lim _{x \rightarrow 1, y \rightarrow-1}\left\{H_{s}^{(0)}(x y) H_{1}^{(1)}(x)-H_{s}^{(0)}(y) H_{1}^{(1)}(x)\right\}=0 .
\end{gathered}
$$

Combining (2.10)-(2.17) and taking $(x, y) \rightarrow(1,1),(1,-1)$, we can obtain $(2.5)$ and (2.6). If taking $(x, y) \rightarrow(1,-1)$ in (2.10)-(2.12) and combining (1.9), we can give the following Corollary.

Corollary 2.3 Let $s \geq 2$ be integer, we have

$$
\begin{aligned}
\sum_{n=1}^{\infty} \frac{\sum_{k=1}^{n-1} \frac{H_{k}}{n-k}(-1)^{k}}{n^{s}}= & \sum_{n=1}^{\infty} \frac{H_{n}^{2}}{n^{s}}(-1)^{n-1}+\frac{1}{2}\left(\zeta(2)-\ln ^{2} 2\right) \bar{\zeta}(s)+\ln 2\left(\zeta^{\star}(\bar{s}, \overline{1})-\zeta^{\star}(\bar{s}, 1)\right) \\
& -\sum_{j=1}^{s-1} \zeta^{\star}(\bar{j}, 1) \zeta(s+1-j)+(s+1) \zeta^{\star}(\overline{s+1}, 1) \\
& -\frac{1}{2} \sum_{n=1}^{\infty} \frac{L_{n}^{2}(1)}{n^{s}}(-1)^{n-1}+\frac{1}{2} \zeta^{\star}(\bar{s}, 2) .
\end{aligned}
$$

In the same way, we can obtain the following Theorem.

Theorem 2.4 Let $s \geq 1$ be integer, then have

$$
\begin{aligned}
\zeta^{\star}(s, \overline{1}, 1)= & -\sum_{n=1}^{\infty} \frac{\sum_{k=1}^{n} \frac{H_{k}}{k}(-1)^{k-1}}{n^{s}} \\
= & s \zeta^{\star}(s+1, \overline{1})-\sum_{n=1}^{\infty} \frac{L_{n}^{2}(1)}{n^{s}}+2 \zeta^{\star}(s, \overline{2})-\ln 2 \zeta^{\star}(s, \overline{1}) \\
& +\zeta^{\star}(\overline{s+1}, \overline{1})+\zeta(s) \zeta^{\star}(\overline{1}, \overline{1})+\sum_{j=1}^{s} \zeta^{\star}(\bar{j}, \overline{1}) \bar{\zeta}(s+1-j), \quad(s \geq 2) . \\
\zeta^{\star}(\bar{s}, \overline{1}, 1)= & \sum_{n=1}^{\infty} \frac{\sum_{k=1}^{n} \frac{H_{k}}{k}(-1)^{k-1}}{n^{s}}(-1)^{n-1}
\end{aligned}
$$




$$
\begin{aligned}
= & (s+1) \zeta^{\star}(\overline{s+1}, \overline{1})+2 \zeta^{\star}(\bar{s}, \overline{2})-\bar{\zeta}(s) \zeta^{\star}(\overline{1}, \overline{1})-\sum_{n=1}^{\infty} \frac{H_{n} L_{n}(1)}{n^{s}}(-1)^{n-1} \\
& -\sum_{j=1}^{s-1} \zeta^{\star}(\bar{j}, \overline{1}) \zeta(s+1-j) . \\
\sum_{n=1}^{\infty} \frac{(-1)^{n-1} \sum_{k=1}^{n-1} \frac{L_{k}(1)}{n-k}}{n^{s}}= & s \zeta^{\star}(\overline{s+1}, \overline{1})+\sum_{n=1}^{\infty} \frac{L_{n}^{2}(1)}{n^{s}}(-1)^{n-1}+\zeta^{\star}(s+1, \overline{1}) \\
& +\sum_{j=2}^{s} \zeta^{\star}(j, \overline{1}) \bar{\zeta}(s+1-j)-\ln 2 \zeta^{\star}(\bar{s}, \overline{1}) \\
& -\ln 2\left(\zeta^{\star}(\bar{s}, \overline{1})-\zeta^{\star}(\bar{s}, 1)\right)+\zeta^{\star}(\overline{s+1}, 1, \overline{1}) .
\end{aligned}
$$

Proof. Similarly to the proof of Theorem 2.2, considering function

$$
T_{s, t}(x, y)=\sum_{\substack{n, m=1 \\ n \neq m}}^{\infty} \frac{L_{n}(1) x^{n} y^{m}}{n^{s} m^{t}(m-n)}
$$

We have the following identity

$$
\begin{aligned}
\sum_{n=1}^{\infty} & \frac{L_{n}(1)}{n^{s}} x^{n} y^{n} \sum_{n=1}^{\infty} \frac{y^{n}}{n}-\sum_{n=1}^{\infty} \frac{L_{n}(1)}{n^{s}} x^{n} \sum_{j=1}^{n-1} \frac{y^{n-j}}{j} \\
& +\sum_{n=1}^{\infty} \frac{x^{n} y^{n}}{n^{s}} \sum_{m=n+1}^{\infty} \frac{L_{m}(1) x^{m-n}}{m-n}-\sum_{n=1}^{\infty} \frac{y^{n}}{n^{s}} \sum_{m=1}^{n-1} \frac{L_{m}(1) x^{m}}{n-m} \\
& =\sum_{j=1}^{s}\left(\sum_{n=1}^{\infty} \frac{L_{n}(1)}{n^{j}} x^{n}\right)\left(\sum_{n=1}^{\infty} \frac{y^{n}}{n^{s+1-j}}\right)-s \sum_{n=1}^{\infty} \frac{L_{n}(1)}{n^{s+1}} x^{n} y^{n} .
\end{aligned}
$$

Noting that

$$
\begin{aligned}
\sum_{m=n+1}^{\infty} \frac{L_{m}(1)}{m-n} x^{m-n} & =\sum_{j=1}^{\infty} \frac{L_{n+j}(1)}{j} x^{j}=\sum_{j=1}^{\infty} \frac{x^{j}}{j}\left\{\sum_{k=1}^{j} \frac{(-1)^{k-1}}{k}+\sum_{k=j+1}^{n+j} \frac{(-1)^{k-1}}{k}\right\} \\
& =\sum_{j=1}^{\infty} \frac{L_{j}(1)}{j} x^{j}+\sum_{k=1}^{n}(-1)^{k-1} \sum_{j=1}^{\infty} \frac{(-x)^{j}}{j(k+j)} \\
& =\sum_{j=1}^{\infty} \frac{L_{j}(1)}{j} x^{j}+\sum_{k=1}^{n} \frac{(-1)^{k-1}}{k} \sum_{j=1}^{\infty}(-x)^{j}\left(\frac{1}{j}-\frac{1}{k+j}\right)
\end{aligned}
$$

and

$$
\lim _{x \rightarrow 1} \sum_{k=1}^{n} \frac{(-1)^{k-1}}{k} \sum_{j=1}^{\infty}(-x)^{j}\left(\frac{1}{j}-\frac{1}{k+j}\right)=\sum_{k=1}^{n} \frac{L_{k}(1)}{k}-\ln 2\left(H_{n}+L_{n}(1)\right) .
$$

Taking $(x, y) \rightarrow(-1,-1),(-1,1),(1,-1)$ and combining $(1.6)(1.7)(2.23)(2.24)$, we can obtain (2.19)-(2.21). Next, we give two explicit relationships which involve alternating double Euler sums and triple linear sums. 
Theorem 2.5 For $p \geq 1$ and $p \in \mathbb{Z}$, we have

$$
\begin{aligned}
\zeta^{\star}(\overline{p+1}, 1, \overline{1})= & \sum_{n=1}^{\infty} \frac{(-1)^{n-1}}{n^{p+1}}\left(\sum_{k=1}^{n} \frac{L_{k}(1)}{k}\right) \\
= & \sum_{i=1}^{p-1}(-1)^{i-1} \zeta(p+1-i) \bar{\zeta}(i+2)-(-1)^{p-1} \zeta^{\star}(\overline{p+2}, 1) \\
& +\ln 2\left(\zeta^{\star}(\overline{p+1}, \overline{1})-\zeta^{\star}(\overline{p+1}, 1)\right)-\frac{1}{2} \ln ^{2} 2\{\zeta(p+1)+\bar{\zeta}(p+1)\} \\
& +\sum_{i=1}^{p-1}(-1)^{i-1} \zeta(p+1-i) \zeta^{\star}(\overline{i+1}, 1) \\
& -(-1)^{p-1} \sum_{n=1}^{\infty} \frac{H_{n}^{2}}{n^{p+1}}(-1)^{n-1} \cdot \\
-\zeta^{\star}(p+1,1, \overline{1})= & \sum_{n=1}^{\infty} \frac{1}{n^{p+1}}\left(\sum_{k=1}^{n} \frac{L_{k}(1)}{k}\right) \\
= & \ln 2\left(\zeta^{\star}(p+1,1)-\zeta^{\star}(p+1, \overline{1})\right)+\sum_{i=1}^{p-1}(-1)^{i-1} \bar{\zeta}(p+1-i) \zeta^{\star}(\overline{i+1}, 1) \\
+ & \ln 2(-1)^{p-1}\{\zeta(p+2)+\bar{\zeta}(p+2)\}-(-1)^{p} \zeta^{\star}(p+2, \overline{1}) \\
& -\frac{1}{2} \ln 2\{\zeta(p+1)+\bar{\zeta}(p+1)\}-\ln 2(-1)^{p-1}\left(\zeta^{\star}(p+1,1)-\zeta^{\star}(\overline{p+1}, 1)\right) \\
& +\sum_{i=1}^{p-1}(-1)^{i-1} \bar{\zeta}(p+1-i) \bar{\zeta}(i+2)-(-1)^{p} \sum_{n=1}^{\infty} \frac{H_{n} L_{n}(1)}{n^{p+1}} .
\end{aligned}
$$

Proof. Using integration by parts, we can verify that

$$
\begin{aligned}
& \int_{0}^{x} t^{n-1} \operatorname{Li}_{q}(t) d t=\sum_{i=1}^{q-1}(-1)^{i-1} \frac{x^{n}}{n^{i}} \operatorname{Li}_{q+1-i}(x)+\frac{(-1)^{q}}{n^{q}} \ln (1-x)\left(x^{n}-1\right)-\frac{(-1)^{q}}{n^{q}}\left(\sum_{k=1}^{n} \frac{x^{k}}{k}\right), \\
& \int_{0}^{x} t^{n-1} \ln ^{2}(1-t) d t=\frac{1}{n}\left(x^{n}-1\right) \ln ^{2}(1-x)-\frac{2}{n} \sum_{k=1}^{n} \frac{1}{k}\left\{x^{k} \ln (1-x)-\sum_{j=1}^{k} \frac{x^{j}}{j}-\ln (1-x)\right\} .
\end{aligned}
$$

Now, we consider the following integral

$$
\int_{0}^{1} \frac{\operatorname{Li}_{p}(x) \ln ^{2}(1+x)}{x} d x, 1 \leq p \in Z
$$

Using (1.12) and (2.28), we can find that

$$
\int_{0}^{1} \frac{\operatorname{Li}_{p}(x) \ln ^{2}(1+x)}{x} d x=2 \sum_{n=1}^{\infty}\left\{\frac{(-1)^{n-1}}{n^{2}}-\frac{H_{n}}{n}(-1)^{n-1}\right\} \int_{0}^{1} x^{n-1} \operatorname{Li}_{p}(x) d x
$$




$$
\begin{aligned}
= & 2 \sum_{n=1}^{\infty}\left\{\frac{(-1)^{n-1}}{n^{2}}-\frac{H_{n}}{n}(-1)^{n-1}\right\}\left\{\sum_{i=1}^{p-1} \frac{(-1)^{i-1}}{n^{i}} \zeta(p+1-i)+\frac{(-1)^{p-1}}{n^{p}} H_{n}\right\} \\
= & 2 \sum_{i=1}^{p-1}(-1)^{i-1} \zeta(p+1-i) \bar{\zeta}(i+2)-2(-1)^{p-1} \zeta^{\star}(\overline{p+2}, 1) \\
& +2 \sum_{i=1}^{p-1}(-1)^{i-1} \zeta(p+1-i) \zeta^{\star}(\overline{i+1}, 1)-2(-1)^{p-1} \sum_{n=1}^{\infty} \frac{H_{n}^{2}}{n^{p+1}}(-1)^{n-1} .
\end{aligned}
$$

On the other hand, by (2.29), we have

$$
\begin{aligned}
& \int_{0}^{1} \frac{\operatorname{Li}_{p}(x) \ln ^{2}(1+x)}{x} d x=\sum_{n=1}^{\infty} \frac{1}{n^{p}} \int_{0}^{1} x^{n-1} \ln ^{2}(1+x) d x \\
& =\ln ^{2} 2\{\zeta(p+1)+\bar{\zeta}(p+1)\}-2 \ln 2\left(\zeta^{\star}(\overline{p+1}, \overline{1})-\zeta^{\star}(\overline{p+1}, 1)\right) \\
& \quad+2 \zeta^{\star}(\overline{p+1}, 1, \overline{1}) .
\end{aligned}
$$

Combining (2.30) and (2.31), we can obtain (2.26). Similarly to the proof of (2.26), considering integral

$$
\int_{0}^{-1} \frac{\operatorname{Li}_{p}(x) \ln ^{2}(1-x)}{x} d x
$$

we deduce (2.27) holds.

\section{Representation of Euler sums by zeta values and linear sums}

In this section, we consider the analytic representations of quadratic Euler sums of the form

$$
\sum_{n=1}^{\infty} \frac{H_{n}^{2}}{n^{2 m}}(-1)^{n-1}, \sum_{n=1}^{\infty} \frac{L_{n}^{2}(1)}{n^{2 m}}(-1)^{n-1}, \sum_{n=1}^{\infty} \frac{H_{n} L_{n}(1)}{n^{2 m}}(-1)^{n-1}, \sum_{n=1}^{\infty} \frac{H_{n} L_{n}(1)}{n^{2 m}}, \sum_{n=1}^{\infty} \frac{L_{n}^{2}(1)}{n^{2 m}}
$$

through zeta values and linear sums, and give explicit formulae for several 6th-order quadratic sums in terms of zeta values and linear sums.

Theorem 3.1 For $m \geq 1$ and $m \in \mathbb{Z}$, we have

$$
\begin{aligned}
\sum_{n=1}^{\infty} \frac{H_{n}^{2}}{n^{2 m}(-1)^{n-1}=} & -\zeta^{\star}(\overline{2 m}, 2)-(2 m+1) \zeta^{\star}(\overline{2 m+1}, 1)-\zeta^{\star}(2 m+1,1) \\
& -\zeta(2) \bar{\zeta}(2 m)-2 \sum_{l=2}^{m} \zeta^{\star}(2 l-1,1) \bar{\zeta}(2 m+2-2 l), \\
\sum_{n=1}^{\infty} \frac{H_{n} L_{n}(1)}{n^{2 m}}(-1)^{n-1}= & \sum_{l=1}^{m-1} \zeta^{\star}(2 l, 1) \bar{\zeta}(2 m+1-2 l)-\ln 2 \zeta^{\star}(\overline{2 m}, 1) \\
& +\ln 2 \zeta^{\star}(2 m, 1)+\frac{1}{2} \zeta^{*}(2 m+1,1)+\zeta^{\star}(\overline{2 m}, 2) \\
& +\frac{2 m-1}{2} \zeta^{\star}(\overline{2 m+1}, 1)-\frac{1}{2} \zeta(2) \bar{\zeta}(2 m) .
\end{aligned}
$$


Proof. Replacing $m$ by $2 m-1$ in (2.1) and taking $s=2 m$ in (2.5), we can obtain (3.1) and (3.2).

Theorem 3.2 For $p \geq 1$ and $p \in \mathbb{Z}$, we have

$$
\begin{aligned}
& \frac{3}{2} \sum_{n=1}^{\infty} \frac{L_{n}^{2}(1)}{n^{p+1}}(-1)^{n-1}-\left(1+(-1)^{p-1}\right) \sum_{n=1}^{\infty} \frac{H_{n}^{2}}{n^{p+1}}(-1)^{n-1} \\
= & \ln 2\left(\zeta^{\star}(\overline{p+1}, \overline{1})-\zeta^{\star}(\overline{p+1}, 1)\right)+\frac{1}{2} \zeta(2)\{\zeta(p+1)+\bar{\zeta}(p+1)\} \\
& +\frac{1}{2} \zeta^{\star}(\overline{p+1}, 2)-\sum_{j=2}^{p}\left(1-(-1)^{j-1}\right) \zeta^{\star}(\bar{j}, 1) \zeta(p+2-j) \\
& -\sum_{j=2}^{p+1} \zeta^{\star}(j, \overline{1}) \bar{\zeta}(p+2-j)+\left(p+2+(-1)^{p-1}\right) \zeta^{\star}(\overline{p+2}, 1)-\zeta^{\star}(p+2, \overline{1}) \\
& \quad-\sum_{i=1}^{p-1}(-1)^{i-1} \zeta(p+1-i) \bar{\zeta}(i+2)-(p+1) \zeta^{\star}(\overline{p+2}, \overline{1})+\ln 2 \zeta^{\star}(\overline{p+1}, \overline{1}) .
\end{aligned}
$$

Proof. From (1.8)(2.18) and (2.21), we obtain

$$
\begin{aligned}
\zeta^{\star}(\bar{s}, 1, \overline{1})= & \sum_{n=1}^{\infty} \frac{(-1)^{n-1}}{n^{s}}\left(\sum_{k=1}^{n} \frac{L_{k}(1)}{k}\right) \\
= & \sum_{n=1}^{\infty} \frac{H_{n}^{2}}{n^{s}}(-1)^{n-1}+2 \ln 2\left(\zeta^{\star}(\bar{s}, \overline{1})-\zeta^{\star}(\bar{s}, 1)\right)-\frac{3}{2} \sum_{n=1}^{\infty} \frac{L_{n}^{2}(1)}{n^{s}}(-1)^{n-1} \\
& -\sum_{j=1}^{s-1} \zeta^{\star}(\bar{j}, 1) \zeta(s+1-j)-\sum_{j=2}^{s} \zeta^{\star}(j, \overline{1}) \bar{\zeta}(s+1-j) \\
& +(s+1) \zeta^{\star}(\overline{s+1}, 1)-s \zeta^{\star}(\overline{s+1}, \overline{1})+\ln 2 \zeta^{\star}(\bar{s}, \overline{1}) \\
& -\zeta^{\star}(s+1, \overline{1})+\frac{1}{2} \zeta^{\star}(\bar{s}, 2)+\frac{1}{2}\left\{\zeta(2)-\ln ^{2} 2\right\} \bar{\zeta}(s) .
\end{aligned}
$$

Taking $s=p+1$ in (3.4) and combining (2.26), we can obtain (3.3).

Theorem 3.3 For $p \geq 1$ and $p \in \mathbb{Z}$, we have

$$
\begin{aligned}
\sum_{n=1}^{\infty} & \frac{L_{n}^{2}(1)}{n^{p+1}}+\left((-1)^{p-1}-1\right) \sum_{n=1}^{\infty} \frac{H_{n} L_{n}(1)}{n^{p+1}} \\
= & \zeta^{\star}(p+1, \overline{2})-\ln 2 \zeta^{\star}(p+1, \overline{1})+\zeta^{\star}(\overline{p+2}, \overline{1})+\frac{1}{2} \zeta(2) \zeta(p+1) \\
& +\sum_{i=1}^{p+1} \zeta^{\star}(\bar{i}, \overline{1}) \bar{\zeta}(p+2-i)+\left(p+1+(-1)^{p}\right) \zeta^{\star}(p+2, \overline{1}) \\
& +\frac{1}{2} \ln ^{2} 2\{2 \zeta(p+1)+\bar{\zeta}(p+1)\}+\ln 2(-1)^{p-1}\left(\zeta^{\star}(p+1,1)-\zeta^{\star}(\overline{p+1}, 1)\right) \\
& -\sum_{i=1}^{p-1}(-1)^{i-1} \bar{\zeta}(p+1-i) \zeta^{\star}(\overline{i+1}, 1)-\ln 2\left(\zeta^{\star}(p+1,1)-\zeta^{\star}(p+1, \overline{1})\right)
\end{aligned}
$$




$$
-\sum_{i=1}^{p-1}(-1)^{i-1} \bar{\zeta}(p+1-i) \bar{\zeta}(i+2)-\ln 2(-1)^{p-1}\{\zeta(p+2)+\bar{\zeta}(p+2)\} .
$$

Proof. The following identity is easily derived

$$
\sum_{k=1}^{n} \frac{H_{k}}{k}(-1)^{k-1}+\sum_{k=1}^{n} \frac{L_{k}(1)}{k}=H_{n} L_{n}(1)+L_{n}(2) .
$$

Multiplying (3.6) by $\frac{1}{n^{p+1}}$ and summing with respect to $n$, the result is

$$
\begin{aligned}
\zeta^{\star}(p+1, \overline{1}, 1)+\zeta^{\star}(p+1,1, \overline{1}) & =-\sum_{n=1}^{\infty} \frac{1}{n^{p+1}}\left(\sum_{k=1}^{n} \frac{H_{k}}{k}(-1)^{k-1}\right)-\sum_{n=1}^{\infty} \frac{1}{n^{p+1}}\left(\sum_{k=1}^{n} \frac{L_{k}(1)}{k}\right) \\
& =-\sum_{n=1}^{\infty} \frac{H_{n} L_{n}(1)}{n^{p+1}}+\zeta^{\star}(p+1, \overline{2}) .
\end{aligned}
$$

Substituting (2.19)(2.27) into (3.7), we deduce Theorem 3.3 holds.

Theorem 3.4 For $l \geq 2, p \geq 1$ and $l, p \in \mathbb{Z}$, we have

$$
\begin{aligned}
& \sum_{n=1}^{\infty} \frac{\zeta_{n}^{2}(p)}{n^{l}}=2^{2 p+l-3}\left\{\sum_{n=1}^{\infty} \frac{\left(\zeta_{n}(p)-L_{n}(p)\right)^{2}}{n^{l}}\left(1-(-1)^{n-1}\right)\right\}, \\
& \sum_{n=1}^{\infty} \frac{\zeta_{n}^{2}(p)}{(2 n+1)^{l}}=2^{2 p-3}\left\{\sum_{n=1}^{\infty} \frac{\left(\zeta_{n}(p)-L_{n}(p)\right)^{2}}{n^{l}}\left(1+(-1)^{n-1}\right)\right\} .
\end{aligned}
$$

Proof. By the definitions of harmonic numbers and alternating harmonic numbers, we have the relations

$$
\begin{gathered}
\zeta_{2 n}(p)-L_{2 n}(p)=\frac{1}{2^{p-1}} \zeta_{n}(p), \zeta_{2 n+1}(p)-L_{2 n+1}(p)=\frac{1}{2^{p-1}} \zeta_{n}(p), \\
\zeta_{2 n}(p)+L_{2 n}(p)=2 h_{n}(p), \zeta_{2 n+1}(p)+L_{2 n+1}(p)=2 h_{n+1}(p) .
\end{gathered}
$$

Here $h_{n}(p)$ is defined by

$$
h_{n}(p)=\sum_{j=1}^{n} \frac{1}{(2 j-1)^{p}} .
$$

Hence, from (3.10) and (3.11), we get

$$
\begin{gathered}
\sum_{n=1}^{\infty} \frac{\left(\zeta_{n}(p)-L_{n}(p)\right)^{2}}{n^{l}}=\frac{1}{2^{2 p+l-2}} \sum_{n=1}^{\infty} \frac{\zeta_{n}^{2}(p)}{n^{l}}+\frac{1}{2^{2 p-2}} \sum_{n=1}^{\infty} \frac{\zeta_{n}^{2}(p)}{(2 n+1)^{l}} \\
\sum_{n=1}^{\infty} \frac{\left(\zeta_{n}(p)-L_{n}(p)\right)^{2}}{n^{l}}(-1)^{n-1}=\frac{1}{2^{2 p-2}} \sum_{n=1}^{\infty} \frac{\zeta_{n}^{2}(p)}{(2 n+1)^{l}}-\frac{1}{2^{2 p+l-2}} \sum_{n=1}^{\infty} \frac{\zeta_{n}^{2}(p)}{n^{l}} .
\end{gathered}
$$

Combining (3.12) and (3.13), we can obtain (3.8) and (3.9). Taking $p=1, l=2 m$ in (3.8) and (3.9), we have

$$
\sum_{n=1}^{\infty} \frac{H_{n} L_{n}(1)}{n^{2 m}}=\left(\frac{1}{2}-\frac{1}{2^{2 m}}\right) \sum_{n=1}^{\infty} \frac{H_{n}^{2}}{n^{2 m}}+\frac{1}{2} \sum_{n=1}^{\infty} \frac{L_{n}^{2}(1)}{n^{2 m}}-\frac{1}{2} \sum_{n=1}^{\infty} \frac{H_{n}^{2}-2 H_{n} L_{n}(1)+L_{n}^{2}(1)}{n^{2 m}}(-1)^{n-1},
$$




$$
\sum_{n=1}^{\infty} \frac{H_{n}^{2}}{(2 n+1)^{2 m}}=\frac{1}{2}\left\{\sum_{n=1}^{\infty} \frac{H_{n}^{2}-2 H_{n} L_{n}(1)+L_{n}^{2}(1)}{n^{2 m}}\left(1+(-1)^{n-1}\right)\right\} .
$$

From (2.6)(3.1)-(3.3)(3.5)(3.14) and (3.15), we have the following Theorem.

Theorem 3.5 For $m \geq 1$ and $m \in Z$, the quadratic sums

$$
\begin{aligned}
& \sum_{n=1}^{\infty} \frac{L_{n}^{2}(1)}{n^{2 m}}(-1)^{n-1}, \sum_{n=1}^{\infty} \frac{H_{n} L_{n}(1)}{n^{2 m}}, \sum_{n=1}^{\infty} \frac{H_{n} L_{n}(1)}{n^{2 m}}(-1)^{n-1} \\
& \sum_{n=1}^{\infty} \frac{H_{n}^{2}}{n^{2 m}}, \sum_{n=1}^{\infty} \frac{H_{n}^{2}}{n^{2 m}}(-1)^{n-1}, \sum_{n=1}^{\infty} \frac{L_{n}^{2}(1)}{n^{2 m}}, \sum_{n=1}^{\infty} \frac{H_{n}^{2}}{(2 n+1)^{2 m}}
\end{aligned}
$$

are reducible to linear sums.

Next, we give some explicit formulae for several 4th and 6th-order quadratic sums in terms of zeta values and linear sums.

Example 1 Some illustrative examples follow.

$$
\begin{aligned}
& \sum_{n=1}^{\infty} \frac{H_{n} L_{n}(1)}{n^{2}}=\frac{43}{16} \zeta(4)+\frac{3}{4} \zeta(2) \ln ^{2} 2-\frac{1}{8} \ln ^{4} 2-3 \operatorname{Li}_{4}\left(\frac{1}{2}\right), \\
& \sum_{n=1}^{\infty} \frac{L_{n}^{2}(1)}{n^{2}}=-\frac{13}{8} \zeta(4)+\frac{5}{2} \zeta(2) \ln ^{2} 2+\frac{1}{12} \ln ^{4} 2+2 \operatorname{Li}_{4}\left(\frac{1}{2}\right), \\
& \sum_{n=1}^{\infty} \frac{H_{n} L_{n}(1)}{n^{2}}(-1)^{n-1}=\frac{29}{16} \zeta(4)+\frac{3}{4} \zeta(2) \ln ^{2} 2-3 \operatorname{Li}_{4}\left(\frac{1}{2}\right)-\frac{1}{8} \ln ^{4} 2, \\
& \sum_{n=1}^{\infty} \frac{H_{n}^{2}}{n^{2}}(-1)^{n-1}=\frac{41}{16} \zeta(4)+\frac{1}{2} \zeta(2) \ln ^{2} 2-\frac{1}{12} \ln ^{4} 2-\frac{7}{4} \zeta(3) \ln 2-2 \operatorname{Li}_{4}\left(\frac{1}{2}\right), \\
& \sum_{n=1}^{\infty} \frac{L_{n}^{2}(1)}{n^{2}}(-1)^{n-1}=-\frac{41}{16} \zeta(4)+2 \zeta(2) \ln ^{2} 2+\frac{1}{6} \ln ^{4} 2+\frac{7}{4} \zeta(3) \ln 2+4 \operatorname{Li}_{4}\left(\frac{1}{2}\right), \\
& \sum_{n=1}^{\infty} \frac{H_{n}^{2}}{(2 n+1)^{2}}=-\frac{61}{16} \zeta(4)+\frac{7}{2} \zeta(2) \ln ^{2} 2+\frac{1}{3} \ln ^{4} 2+8 \operatorname{Li}_{4}\left(\frac{1}{2}\right) \text {. }
\end{aligned}
$$

Using mathematica, the numerical values of 6th-order quadratic sums and 6th-order linear sums, to 30 decimal digits, are:

$$
\begin{aligned}
& \sum_{n=1}^{\infty} \frac{H_{n}}{n^{5}}(-1)^{n-1}=-\zeta^{\star}(\overline{5}, 1)=0.959151942504318157165421137321 \ldots \\
& \sum_{n=1}^{\infty} \frac{L_{n}(1)}{n^{5}}=-\zeta^{\star}(5, \overline{1})=1.02005194570145237930331996837 \ldots \\
& \sum_{n=1}^{\infty} \frac{L_{n}(1)}{n^{5}}(-1)^{n-1}=\zeta^{\star}(\overline{5}, \overline{1})=0.987441426403299713771650007985 \ldots \\
& \sum_{n=1}^{\infty} \frac{\zeta_{n}(2)}{n^{4}}(-1)^{n-1}=-\zeta^{\star}(\overline{4}, 2)=0.934707899349253255197542851216 \ldots
\end{aligned}
$$




$$
\begin{aligned}
& \sum_{n=1}^{\infty} \frac{L_{n}(2)}{n^{4}}=-\zeta^{\star}(4, \overline{2})=1.06358224101814909880154833539 \ldots \\
& \sum_{n=1}^{\infty} \frac{H_{n}^{2}}{n^{4}}(-1)^{n-1}=0.889343140860204925167721149031 \ldots \\
& \sum_{n=1}^{\infty} \frac{L_{n}^{2}(1)}{n^{4}}(-1)^{n-1}=0.992525582596587194230080495889 \ldots \\
& \sum_{n=1}^{\infty} \frac{L_{n}^{2}(1)}{n^{4}}=1.02740836872025737362228676589 \ldots \\
& \sum_{n=1}^{\infty} \frac{H_{n} L_{n}(1)}{n^{4}}=1.07692404770840527955071456146 \ldots \\
& \sum_{n=1}^{\infty} \frac{H_{n} L_{n}(1)}{n^{4}}(-1)^{n-1}=0.969581748999225091836494168654 \ldots \\
& \sum_{n=1}^{\infty} \frac{L_{n}(1) \zeta_{n}(2)}{n^{3}}=1.15935334356951415975457027807 \ldots
\end{aligned}
$$

From Theorem 3.1-3.4, we find that the 6th-order quadratic sums satisfies a relation involving homogeneous combinations of $\zeta(2), \zeta(3), \zeta(4), \zeta(5), \zeta(6), \ln 2$ and 6th-order linear sums.

\section{Example 2}

$$
\begin{aligned}
& \sum_{n=1}^{\infty} \frac{H_{n}^{2}}{n^{4}}(-1)^{n-1}=-\frac{175}{32} \zeta(6)+\frac{1}{2} \zeta^{2}(3)-\zeta^{\star}(\overline{4}, 2)-5 \zeta^{\star}(\overline{5}, 1), \\
& \sum_{n=1}^{\infty} \frac{L_{n}^{2}(1)}{n^{4}}=\frac{15}{4} \ln ^{2} 2 \zeta(4)+\frac{9}{4} \zeta(2) \zeta(3) \ln 2-\frac{31}{4} \zeta(5) \ln 2+\frac{231}{64} \zeta(6)-\frac{45}{32} \zeta^{2}(3) \\
& +\zeta^{\star}(\overline{5}, \overline{1})+\zeta^{\star}(4, \overline{2}), \\
& \sum_{n=1}^{\infty} \frac{L_{n}^{2}(1)}{n^{4}}(-1)^{n-1}=\frac{15}{4} \ln ^{2} 2 \zeta(4)+\frac{9}{4} \zeta(2) \zeta(3) \ln 2-\frac{93}{16} \zeta(5) \ln 2+\frac{35}{64} \zeta(6)-\frac{15}{16} \zeta^{2}(3) \\
& -\zeta^{\star}(\overline{4}, 2), \\
& \sum_{n=1}^{\infty} \frac{H_{n} L_{n}(1)}{n^{4}}=-\frac{3}{2} \zeta(2) \zeta(3) \ln 2+\frac{31}{8} \zeta(5) \ln 2+\frac{2359}{384} \zeta(6)-\frac{7}{64} \zeta^{2}(3)+\frac{1}{2} \zeta^{\star}(\overline{5}, \overline{1}) \\
& +\frac{1}{2} \zeta^{\star}(4, \overline{2})+2 \zeta^{\star}(\overline{4}, 2)+4 \zeta^{\star}(\overline{5}, 1), \\
& \sum_{n=1}^{\infty} \frac{H_{n} L_{n}(1)}{n^{4}}(-1)^{n-1}=-\frac{3}{2} \zeta(2) \zeta(3) \ln 2+\frac{155}{32} \zeta(5) \ln 2+\frac{7}{64} \zeta(6)+\frac{5}{4} \zeta^{2}(3) \\
& +\zeta^{\star}(\overline{4}, 2)+\frac{3}{2} \zeta^{\star}(\overline{5}, 1) .
\end{aligned}
$$

On the other hand, by Cauchy product formula, we have

$$
\mathrm{Li}_{2}^{2}(x)=2 \sum_{n=1}^{\infty}\left\{\frac{\zeta_{n}(2)}{n^{2}}+2 \frac{H_{n}}{n^{3}}\right\} x^{n}-6 \mathrm{Li}_{4}(x) .
$$


Multiplying (3.16) by $\frac{\ln (1-x)}{x}$, and integrating over $(0, x)$. The result is

$$
\mathrm{Li}_{2}^{3}(x)=\sum_{n=1}^{\infty}\left\{\frac{18}{n^{5}}-6\left[\frac{\zeta_{n}(2)}{n^{3}}+2 \frac{H_{n}}{n^{4}}\right]\right\}\left\{x^{n} \ln (1-x)-\sum_{k=1}^{n} \frac{x^{k}}{k}-\ln (1-x)\right\} .
$$

Taking $x=-1$ in (3.17), using Example 2, we obtain

$$
\begin{aligned}
\sum_{n=1}^{\infty} \frac{L_{n}(1) \zeta_{n}(2)}{n^{3}}= & \frac{29}{8} \zeta(2) \zeta(3) \ln 2-\frac{93}{32} \zeta(5) \ln 2-\frac{1855}{128} \zeta(6)+\frac{17}{16} \zeta^{2}(3)-\zeta^{\star}(\overline{5}, \overline{1}) \\
& -\zeta^{\star}(4, \overline{2})-4 \zeta^{\star}(\overline{4}, 2)-8 \zeta^{\star}(\overline{5}, 1) .
\end{aligned}
$$

Acknowledgments. The authors would like to thank the anonymous referee for his/her helpful comments, which improve the presentation of the paper.

\section{References}

[1] George E. Andrews, Richard Askey, Ranjan Roy. Special Functions. Cambridge University Press., 2000: 481-532.

[2] David H. Bailey, Jonathan M. Borwein and Roland Girgensohn. Experimental evaluation of Euler sums. Experimental Mathematics., 1994, 3(1): 17-30.

[3] David H. Bailey, Jonathan M. Borwein, Richard E. Crandall. Computation and theory of extended Mordell-Tornheim-Witten sums. Math. Comp., 2014, 83(288): 1795-1821.

[4] B. C. Berndt. Ramanujans Notebooks, Part I. Springer-Verlag, New York., 1985.

[5] B. C. Berndt. Ramanujans Notebooks, Part II. Springer-Verlag, New York., 1989.

[6] David Borwein, Jonathan M. Borwein and Roland Girgensohn. Explicit evaluation of Euler sums. Proc. Edinburgh Math., 1995, 38: 277-294.

[7] J.Borwein, P.Borwein, R.Girgensohn, S.Parnes. Making sense of experimental mathematics. Mathematical Intelligencer., 1996, 18(4): 12-18.

[8] Jonathan M. Borwein, David M. Bradley, David J. Broadhurst, Petr. Lisonk. Special values of multiple polylogarithms. Trans. Amer. Math. Soc., 2001, 353(3): 907-941.

[9] J. M. Borwein, I. J. Zucker, J. Boersma. The evaluation of character Euler double sums. Ramanujan J., 2008, 15 (3): 377-405.

[10] J.M. Borwein, R. Girgensohn, Evaluation of triple Euler sums, Electron. J. Combin., 1996: 2-7.

[11] Minking Eie, Chuan-Sheng Wei. Evaluations of some quadruple Euler sums of even weight. Functions et Approximatio., 2012, 46(1): 63-67.

[12] Philippe Flajolet and Bruno Salvy. Euler sums and contour integral representations. Experimental Mathematics., 1998, 7(1): 15-35.

[13] Pedro freitas. Integrals of polylogarithmic functions, recurrence relations, and associated Euler sums. Mathematics of Computation., 2005, 74(251): 1425-1440.

[14] Comtet L. Advanced combinatorics, Boston: D Reidel Publishing Company., 1974.

[15] I. Mezö. Nonlinear Euler sums. Pacific J. Math., 2014, 272: 201-226.

[16] A. Sofo. Quadratic alternating harmonic number sums. J. Number Theory., 2015, 154: 144-159. 
[17] C. Markett. Triple sums and the Riemann zeta function. J. Number Theory., 1994, 48(2): 113-132.

[18] N. Wakabayashi. On hoffman's relation for multiple zeta-star values. International Journal of Number Theory., 2012, 08(8): 1971-1976.

[19] Ce Xu, Jinfa Cheng. Some Results On Euler Sums. Functions et Approximatio., 2016, in press.

[20] Ce Xu, Yuhuan Yan, Zhijuan Shi. Euler sums and integrals of polylogarithm functions. J. Number Theory., 2016, 165: 84-108. 